\title{
Estudo comparativo entre artrotomografia computadorizada multi-slice e artroscopia na avaliação das lesões do manguito rotador*
}

\section{Comparative Study between Multi-slice Computed Tomographic Arthrography and Arthroscopy in the Evaluation of Rotator Cuff Tears}

\author{
Luis Alfredo Gómez-Vieira1® Nicolas Gerardo Gómez-Cordero ${ }^{2}$ \\ ${ }^{1}$ Serviço de Cirurgia de Ombro e Cotovelo, Hospital Português da \\ Bahia, Salvador, BA, Brasil \\ 2 Serviço de Ortopedia, Hospital Português da Bahia, Salvador, BA, \\ Brasil \\ ${ }^{3}$ Curso Superior de Tecnologia em Radiologia, Instituto Federal de \\ Educação, Ciência e Tecnologia da Bahia (IFBA), Salvador, BA, Brasil \\ ${ }^{4}$ Escola Bahiana de Medicina e Saúde Pública (EBMSP), Salvador, BA, \\ Brasil
}

Paulo Mauricio Almeida Geambastiani ${ }^{3}$ Marcos Almeida Matos ${ }^{4}$

Endereço para correspondência Luis Alfredo Gómez-Vieira, Serviço de Cirurgia de Ombro e Cotovelo, Hospital Português da Bahia, Rua do Benjoim, 47, Caminho das Arvores, Salvador, BA, 41820-340, Brasil (e-mail: gomezvieira@hotmail.com).

Rev Bras Ortop 2019;54:579-586.

\section{Resumo}

Palavras-chave

- artrografia/métodos

- manguito rotador

- lesões do ombro/ classificação

- artroscopia
Objetivo Comparar os achados por imagem das alterações anatômicas da artrotomografia computadorizada multi-slice na avaliação das lesões do manguito rotador do ombro e correlacioná-los com os achados da artroscopia (exame diagnóstico padrãoouro).

Materiais e Métodos Estudo longitudinal, prospectivo, comparativo de acurácia diagnóstica, feito de junho de 2016 a junho de 2017, em pacientes de ambos os sexos, com idades entre 40 e 70 anos, com lesão dos tendões do manguito rotador do ombro, e que tinham necessidade terapêutica de fazer artroscopia do ombro. Foram incluídos pacientes com contraindicação à realização de ressonância magnética. Após a artrotomografia computadorizada multi-slice, todos os pacientes foram submetidos a artroscopia.

Resultados Para a obtenção dos resultados, os seguintes parâmetros foram considerados: sensibilidade, especificidade, acurácia, valor preditivo positivo, valor preditivo negativo, coeficiente Kappa, e contraposição do método de imagem com a artroscopia. Conclusão $\mathrm{Na}$ impossibilidade da realização da ressonância magnética (exame de imagem padrão-ouro), a artrotomografia computadorizada multi-slice se mostra um exame de imagem capaz de avaliar/diagnosticar as lesões do manguito rotador.

Trabalho desenvolvido no Hospital Português da Bahia, Salvador, BA, Brasil. Publicado Originalmente por Elsevier Ltda.

recebido

16 de Outubro de 2017 aceito

14 de Dezembro de 2017
DOI https://doi.org/

10.1016/j.rbo.2017.12.020. ISSN 0102-3616.
Copyright $\odot 2019$ by Sociedade Brasileira License terms de Ortopedia e Traumatologia. Published by Thieme Revnter Publicações Ltda, Rio de Janeiro, Brazil 


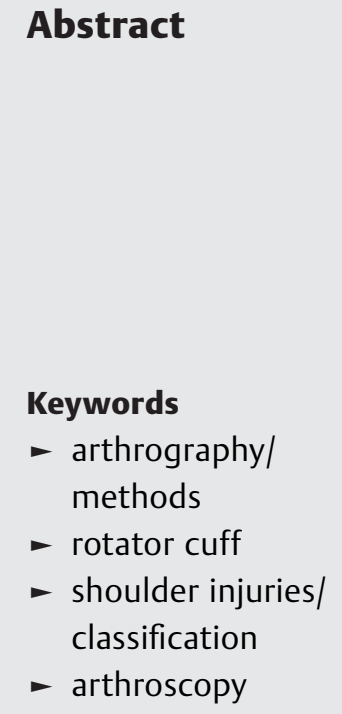

Objective To compare the imaging findings of anatomical alterations using multi-slice computed tomographic arthrography in the evaluation of rotator cuff tears of the shoulder, correlating them with the arthroscopy (the gold standard diagnostic test) findings.

Materials and Methods A longitudinal, prospective, comparative study of diagnostic accuracy performed in the period between June 2016 and June 2017 in patients of both sexes, aged between 40 and 70 years, with shoulder rotator cuff tendon tears and therapeutic need to undergo shoulder arthroscopy. Patients with contraindication to magnetic resonance imaging were included. After multi-slice computed tomographic arthrography, all patients underwent arthroscopy.

Results To obtain the results, the following parameters were determined: sensitivity, specificity, accuracy, positive predictive value, negative predictive value, Kappa coefficient, and contrast between the imaging method and arthroscopy.

Conclusion In the impossibility of performing magnetic resonance imaging (the gold standard imaging technique), multi-slice computed tomographic arthrography is an imaging examination capable of evaluating/diagnosing rotator cuff tears.

\section{Introdução}

As lesões que afetam o manguito rotador estão entre as mais frequentes causas de dor no ombro, com probabilidade de gerar marcante impotência funcional. ${ }^{1-3}$ Essas lesões representam um espectro de doenças que vão desde tendinite aguda até lesão extensa, e comprometem todos os componentes anatômicos do ombro. Além de alta prevalência, que varia de $7 \%$ a $40 \%$, sabe-se também que essa desordem aumenta em proporção direta com o avançar da idade. ${ }^{4}$

O manguito rotador atua para estabilizar dinamicamente e equilibrar a cabeça do úmero em relação à glenoide, enquanto o grupo muscular axial (deltoide e peitoral maior etc.) age para mover o úmero: a ruptura do manguito rotador pode levar facilmente à perda da função do $\mathrm{mbro}^{5,6} \mathrm{em}$ variados graus.

A idade do paciente, os sintomas e o nível de atividade influenciam diretamente no tratamento, assim como a presença de outras anormalidades associadas, tais como lesões labrais, de cartilagem e ósseas. ${ }^{7-9}$

Na avaliação do manguito rotador, o exame de ressonância magnética (RM) é considerado o padrão-ouro em comparação com os demais métodos de diagnóstico por imagem. Trata-se de um exame que de captação de ondas eletromagnéticas para a definição de imagens do corpo humano. ${ }^{10}$ Algumas sequências durante o exame, entretanto, tornam a RM relativamente demorada, o que se torna um problema sério naqueles pacientes com "traços" de comportamento claustrofóbico ou, de fato, claustrofóbicos. Além disso, pacientes portadores de implantes metálicos podem ter contraindicação para esse exame. Alteração do funcionamento de marca-passo cardíaco ou mesmo deslocamento de clipes cerebrais e implantes ortopédicos são exemplos desse tipo de preocupação.

Assim, em virtude da inexistência, até o momento, de um exame imagiológico opcional no diagnóstico da lesão do manguito rotador do ombro, o objetivo deste trabalho é comparar a eficácia da artrotomografia computadorizada multi-slice (ATCMS) na lesão do manguito rotador do ombro, em um grupo de pacientes com contraindicação relativa ou absoluta para o exame de RM, e os achados por imagem das alterações anatômicas da ATCMS com os achados artroscópicos na avaliação das lesões do manguito rotador do ombro.

\section{Materiais e Métodos}

Estudo longitudinal, prospectivo, comparativo, feito de junho de 2016 a junho de 2017, em pacientes de ambos os sexos, com idades entre 40 e 70 anos, com histórico e achados clínicos de lesão dos tendões do manguito rotador do ombro (testes de Jobe, Patte, e Gerber positivos), e que tinham necessidade clínica e terapêutica de fazer artroscopia do ombro por não responderem ao tratamento conservador (medicação analgésica e anti-inflamatória, fisioterapia, hidroterapia, infiltração etc.).

Foram incluídos pacientes originários do Serviço de Ortopedia da nossa instituição com contraindicação à RM, e cujo médico assistente tinha solicitado exame de artrotomografia computadorizada do ombro como método opcional de avaliação.

Os critérios de exclusão foram: ter feito procedimentos cirúrgicos prévios no ombro acometido, incapacidade de responder ao questionário clínico ou de compreender o termo de consentimento livre e esclarecido (TCLE), ou não estivar acompanhado de responsável capaz de fazê-lo; também foram excluídos pacientes portadores de alergias, claustrofóbicos, com hipertireoidismo em atividade, insuficiência cardíaca grave, insuficiência pulmonar de alto grau, asma, insuficiência renal, doenças autoimunes, mieloma múltiplo, nefropatias com diabetes mellitus, ou outras patologias graves não relacionadas ao objetivo do estudo.

Todos os pacientes elegíveis para o estudo foram adequadamente informados dos objetivos, riscos e benefícios da pesquisa, e entraram no protocolo após estar devidamente conscientes do seu desejo e após assinar o TCLE. A pesquisa só teve início após apreciação e aprovação do protocolo pelo Comitê de Ética em Pesquisa da instituição. 
Os pacientes foram encaminhados para o Setor de Tomografia Computadorizada para exames de ATCMS conforme protocolo clinicamente padronizado. Posteriormente, todos os pacientes com indicação de tratamento cirúrgico artroscópico foram submetidos à artroscopia da maneira convencional, e sem a interferência dos pesquisadores na indicação ou no protocolo dos procedimentos. A avaliação ortopédica foi feita por meio de pesquisa do histórico e dos achados clínicos de lesão do manguito rotador de acordo com os critérios adotados pelo médico assistente. Os achados clínicos diagnósticos foram registrados em fichas de exame próprias, que serviram para o planejamento do tratamento da lesão do manguito rotador no ombro do paciente estudado.

A ATCMS de cada paciente foi feita em um único dia com a técnica convencional no aparelho Optima (GE, Chicago, IL, EUA), com 128 multidetectores. Previamente ao método, os pacientes foram submetidos a artrografia por meio dos seguintes passos: a) identificação do ponto exato onde seria introduzida a agulha intra-articular por fluoroscopia; b) assepsia e antissepsia do ombro a ser examinado com clorexedina a $2 \%$ (degermante) e clorexedina a $0,5 \%$ (solução alcoólica); c) anestesia local com xilocaína a $2 \%$ introduzida por meio de agulha $30 \times 7 \mathrm{~mm}$; d) introdução de agulha de raquianestesia de 18 ou $20 \mathrm{Ga}$, de $7 \mathrm{~cm}$, até alcançar a articulação glenoumeral, guiada sob visão fluoroscópica; e) injeção na articulação glenoumeral de 10 a $12 \mathrm{~mL}$ de solução com $5 \mathrm{~mL}$ de meio de contraste diluído em $100 \mathrm{~mL}$ de soro fisiológico a $0,9 \%$, guiada pela fluoroscopia da tomografia computadorizada pela via anterior; ef) encaminhamento dos pacientes para exame de imagem.

Após a artroscopia no paciente, todas as imagens de ATCMS foram analisadas novamente por radiologistas com mais de 10 anos de experiência em radiologia musculoesquelética, e que não tiveram acesso ao histórico clínico ou aos resultados da artroscopia. Para prevenir falhas, os autores analisaram separadamente todas as ATCMS sem identificação dos pacientes.

As artroscopias foram feitas da forma convencional, de acordo com a técnica, protocolo e decisão do médico assistente. Foram feitos três pequenos cortes para a confecção dos portais artroscópicos (anterior, lateral e posterior) no ombro do paciente. No portal posterior ficava a câmera para filmagem e visualização da cirurgia, e os portais anterior e lateral serviram para introdução do material cirúrgico necessário para a correção das lesões do manguito rotador. Após o procedimento, os autores do presente trabalho tiveram acesso aos vídeos das artroscopias para análise das lesões anatômicas existentes em cada paciente, com registro delas em protocolo próprio.

Como exame padrão-ouro, consideramos a artroscopia, e foram determinados os seguintes parâmetros: sensibilidade, especificidade, acurácia, valor preditivo positivo, valor preditivo negativo, e coeficiente Kappa, com contraposição do método de imagem (ATCMS) com a artroscopia. Nesse intuito, foram construídas tabelas de dupla entrada do tipo $2 \times 2$, com frequências e percentuais dos pares de variáveis formados e seus respectivos valores marginais e totais; com isso, para cada tabela construída, foram calculadas as medidas resumo supramencionadas. O coeficiente Kappa classi-
Tabela 1 Variáveis sociodemográficas e clínicas dos pacientes

\begin{tabular}{|l|l|}
\hline Características & $\begin{array}{l}\text { n (\%) ou média } \\
\text { (desvio padrão) }\end{array}$ \\
\hline Sexo & \\
\hline Masculino & $15(50 \%)$ \\
\hline Feminino & $15(50 \%)$ \\
\hline Idade & $57,00(8,28)$ \\
\hline Peso & $68,00(5,56)$ \\
\hline Altura & $1,64(0,06)$ \\
\hline Doença cardiovascular & $16(53,3 \%)$ \\
\hline Uso de marca-passo & $9(30 \%)$ \\
\hline Outras comorbidades & $19(63,3 \%)$ \\
\hline Claustrofobia & $18(60 \%)$ \\
\hline Presença de implantes metálicos & $16(53,3 \%)$ \\
\hline Membro dominante operado & $18(60 \%)$ \\
\hline Tempo de diagnóstico & \\
\hline Até 6 meses & $2(6,7 \%)$ \\
\hline Mais de 6 e até 12 meses & $12(40 \%)$ \\
\hline Mais de 12 meses & $16(53,3 \%)$ \\
\hline
\end{tabular}

fica o grau de concordância em: nulo (valores menores do que 0), ruim (0-19), razoável (20-39), moderado (40-59), substancial (60-79), e quase perfeito (80-100). ${ }^{11}$

As variáveis sociodemográficas e clínicas dos pacientes, assim como os achados na ATCMS e na artroscopia, estão resumidas nas $\mathbf{-}$ Tabelas $\mathbf{1 - 5}$.

A distribuição por gênero neste estudo, por mero acaso, foi exatamente igual, com 15 homens e 15 mulheres, com média de idade de 57 anos, peso médio de $68 \mathrm{Kg}$, e altura média de $1,64 \mathrm{~m}$. 0 membro dominante dos pacientes foi operado em $60 \%$ dos casos, e o diagnóstico da lesão, na maioria dos casos (53,3\%), levou mais de 12 meses para ser estabelecido.

Tabela 2 Lesões encontradas na artrotomografia computadorizada e na artroscopia

\begin{tabular}{|l|l|l|l|}
\hline $\begin{array}{l}\text { Artrotomografia } \\
\text { computadorizada }\end{array}$ & $\begin{array}{l}\text { Com lesão } \\
\text { artroscópica }\end{array}$ & $\begin{array}{l}\text { Sem lesão } \\
\text { artroscópica }\end{array}$ & $p$ \\
\hline Manguito rotador & & & \\
\hline Com lesão & 27 & 0 & \\
\hline Sem lesão & 7 & 4 & \\
\hline Supraespinal & & & \\
\hline Com lesão & 26 & 0 & $<0,001$ \\
\hline Sem lesão & 7 & 5 & \\
\hline Infraespinal & & & $<0,001$ \\
\hline Com lesão & 15 & 0 & \\
\hline Sem lesão & 4 & 19 & \\
\hline Subescapular & & & \\
\hline Com lesão & 13 & 0 & \\
\hline Sem lesão & 3 & 22 & \\
\hline
\end{tabular}


582 Estudo comparativo entre artrotomografia computadorizada multi-slice e artroscopia Gómez-Vieira et al.

Tabela 3 Características das lesões encontradas na artrotomografia computadorizada e na artroscopia

\begin{tabular}{|l|l|l|}
\hline Lesões & ATC n (\%) & Artroscopia n (\%) \\
\hline Supraespinal & & \\
\hline Intacta & $4(13,3 \%)$ & 0 \\
\hline Completa & $12(40 \%)$ & $15(50 \%)$ \\
\hline Transfixante & $9(30 \%)$ & $6(20 \%)$ \\
\hline Bursal parcial & $1(3,33 \%)$ & $5(16,7 \%)$ \\
\hline Intrasubstancial parcial & 0 & 0 \\
\hline Articular parcial & $4(13,3 \%)$ & $4(13,3 \%)$ \\
\hline Infraespinal & & \\
\hline Intacta & $15(50 \%)$ & $13(43,33 \%)$ \\
\hline Completa & $6(20 \%)$ & $8(26,67 \%)$ \\
\hline Transfixante & $1(3,33 \%)$ & $1(3,33 \%)$ \\
\hline Bursal parcial & 0 & $1(3,33 \%)$ \\
\hline Intrasubstancial parcial & 0 & 0 \\
\hline Articular parcial & $8(26,67 \%)$ & $7(23,33 \%)$ \\
\hline Subescapular & & \\
\hline Intacta & $17(56,67 \%)$ & $15(50 \%)$ \\
\hline Completa & $5(16,7 \%)$ & $4(13,3 \%)$ \\
\hline Transfixante & $1(3,33 \%)$ & $1(3,33 \%)$ \\
\hline Bursal parcial & 0 & 0 \\
\hline Intrasubstancial parcial & 0 & $1(3,33 \%)$ \\
\hline Articular parcial & $7(23,33 \%)$ & $9(30 \%)$ \\
\hline
\end{tabular}

Abreviatura: ATC, artrotomografia computadorizada.

Tabela 4 Achados associados às lesões do manguito rotador na artrotomografia computadorizada multi-slice e na artroscopia

\begin{tabular}{|l|l|l|}
\hline Achados & ATCMS & Artroscopia \\
\hline $\begin{array}{l}\text { Cabo longo do tendão } \\
\text { do bíceps (lesão) }\end{array}$ & 7 & 8 \\
\hline Âncora do bíceps (lesão) & 0 & 0 \\
\hline Labrum (lesão) & 3 & 3 \\
\hline Cartilagem (lesão) & 2 & 2 \\
\hline Estruturas ósseas (lesão) & 11 & 11 \\
\hline Cápsula articular (lesão) & 0 & 0 \\
\hline Corpo livre (intra-articular) & 0 & 0 \\
\hline
\end{tabular}

Abreviatura: ATCMS, artrotomografia computadorizada multi-slice.

As variáveis clínicas observadas nos pacientes incluíram desde claustrofobia (60\%) a doenças cardiovasculares (53,3\%), além de haver portadores de implantes metálicos $(53,3 \%)$ e de comorbidades diversas $(63,3 \%)$.

\section{Resultados}

As anormalidades mais comumente visualizadas na artroscopia foram as lesões do tendão do músculo supraespinal, evidentes, também, em todos os casos deste estudo na ATCMS; dessas, a mais comumente encontrada foi a lesão completa do supraespinal, evidente em 12 e em 15 pacientes pela ATCMS e pela artroscopia, respectivamente (-Fig. 1).

Quanto às segundas lesões mais comuns - as do tendão músculo infraespinal -, a mais comumente encontrada foi a lesão parcial articular, evidente em oito pacientes pela ATCMS, e em sete pacientes pela artroscopia.

Em 27 pacientes, a artroscopia detectou lesões do manguito rotador. Já a ATCMS detectou a lesão em 20 pacientes.

Das lesões do manguito rotador, a artroscopia constatou lesão do supraespinal em 26 pacientes, do infraespinal em 15, e do subescapular em 13, enquanto, na ATCMS, a distribuição dessas lesões se deu em 20,11 e 10 pacientes, respectivamente.

As lesões foram distinguidas em: lesão completa, lesão transfixante, lesão parcial bursal, lesão parcial intrasubstancial e lesão parcial articular.

Na ATCMS, as lesões do tendão supraespinal em 12 pacientes eram do tipo completo; 9 pacientes tinham lesões transfixantes; apenas um pasciente tinha lesão parcial bursal; e quatro tinham lesão parcial articular.Já as lesões do tendão infraespinal se distribuíram da seguinte forma: completa em seis pacientes; transfixante em um; e parcial articular em oito. E as lesões do tendão subescapular estavam distribuídas da seguinte maneira: cinco pacientes com lesão completa; lesão transfixante em um; e lesão parcial articular em sete (-Figs. 2 e 3 ).

$\mathrm{Na}$ artroscopia, as lesões do tendão supraespinal eram: completas em cinco pacientes; transfixantes em seis; parciais bursais em cinco; e lesão parciais articulares em quatro pacientes. Já as lesões do tendão infraespinal se distribuíram da seguinte forma: lesão completa em oito pacientes; transfixante em um; parcial bursal em um; e parcial articular em sete. $E$ as lesões do tendão estavam distribuídas da seguinte maneira: completa em quatro pacientes; transfixante em um; parcial intrasubstancial em um; e parcial articular em nove.

Quanto aos achados associados às lesões do manguito rotador, as lesões labrais (três pacientes), as cartilaginosas (dois pacientes ) e as ósseas (onze pacientes) foram igualmente identificadas tanto na ATCMS quanto na artroscopia. Quanto à lesão associada do cabo longo do tendão do bíceps, na artroscopia, ela foi identificada em oito pacientes, enquanto na ATCMS foi identificada em sete.

A lesão parcial intrasubstancial dos tendões supraespinal e infraespinal não foi encontrada por meio da ATCMS ou da artroscopia. Ela foi identificada apenas uma vez no tendão subescapular pela artroscopia.

As lesões na âncora do bíceps e na cápsula articular, assim como a presença de corpo livre articular, não foram identificadas pela artroscopia nem pela ATCMS.

No presente estudo, para efeito de cálculo de sensibilidade e especificidade, se fez necessário pegar uma amostra em um grupo distinto de oito pacientes com outras patologias que não lesão do manguito rotador, e que também foram submetidos a ATCMS e artroscopia.

Assim, a nossa especificidade (pacientes não doentes) não se reflete em absoluto em função do seu viés de seleção. No entanto, a sensibilidade (pacientes doentes) representa perfeitamente a realidade. 
Tabela 5 Artrotomografia computadorizada multi-slice - sensibilidade, especificidade, valor preditivo positivo, valor preditivo negativo, acurácia e coeficiente Kappa (\%)

\begin{tabular}{|l|l|l|l|l|}
\hline Variável & Manguito & Supraespinal & Infraespinal & Subescapular \\
\hline Sensibilidade & $79,41(\%)$ & $78,78(\%)$ & $78,95(\%)$ & $81,25(\%)$ \\
\hline Especificidade & $100(\%)$ & $100(\%)$ & $100(\%)$ & $100(\%)$ \\
\hline VPP & $100(\%)$ & $100(\%)$ & $100(\%)$ & $100(\%)$ \\
\hline VPN & $36,36(\%)$ & $41,67(\%)$ & $82,61(\%)$ & $88,00(\%)$ \\
\hline Acurácia & $81,57(\%)$ & $81,57(\%)$ & $89,47(\%)$ & $92,11(\%)$ \\
\hline Kappa & $44,44(\%)$ & $49,51(\%)$ & $78,94(\%)$ & $83,39(\%)$ \\
\hline
\end{tabular}

Abreviaturas: VPN, valor preditivo negativo; VPP, valor preditivo positivo.

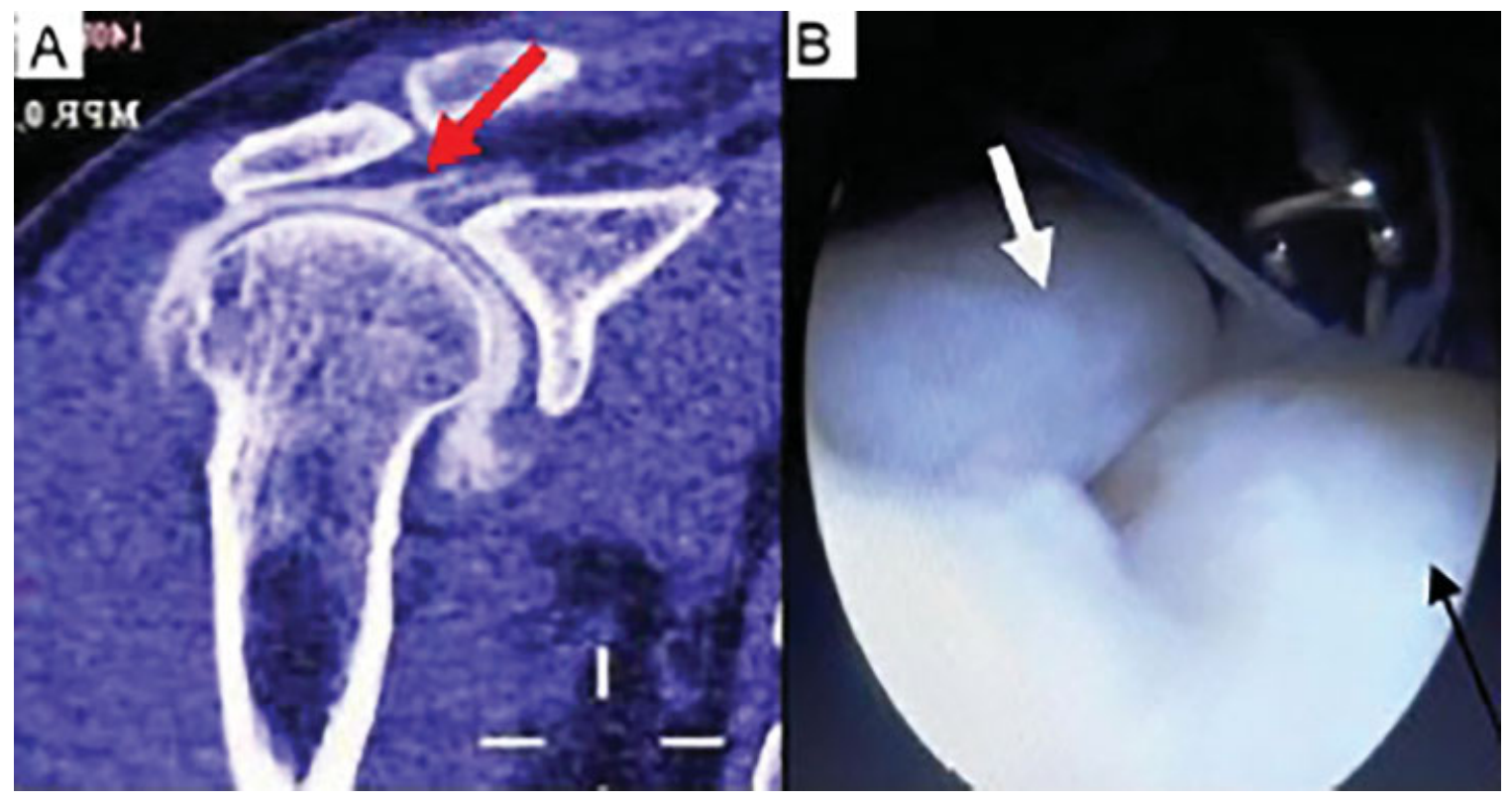

Fig. 1 Lesão completa do tendão supraespinal (seta vermelha). A, corte coronal da artrotomografia computadorizada multi-slice (ATCMS); B, visão artroscópica subacromial (seta branca - tendão supraespinal; seta preta - tuberosidade maior do úmero).

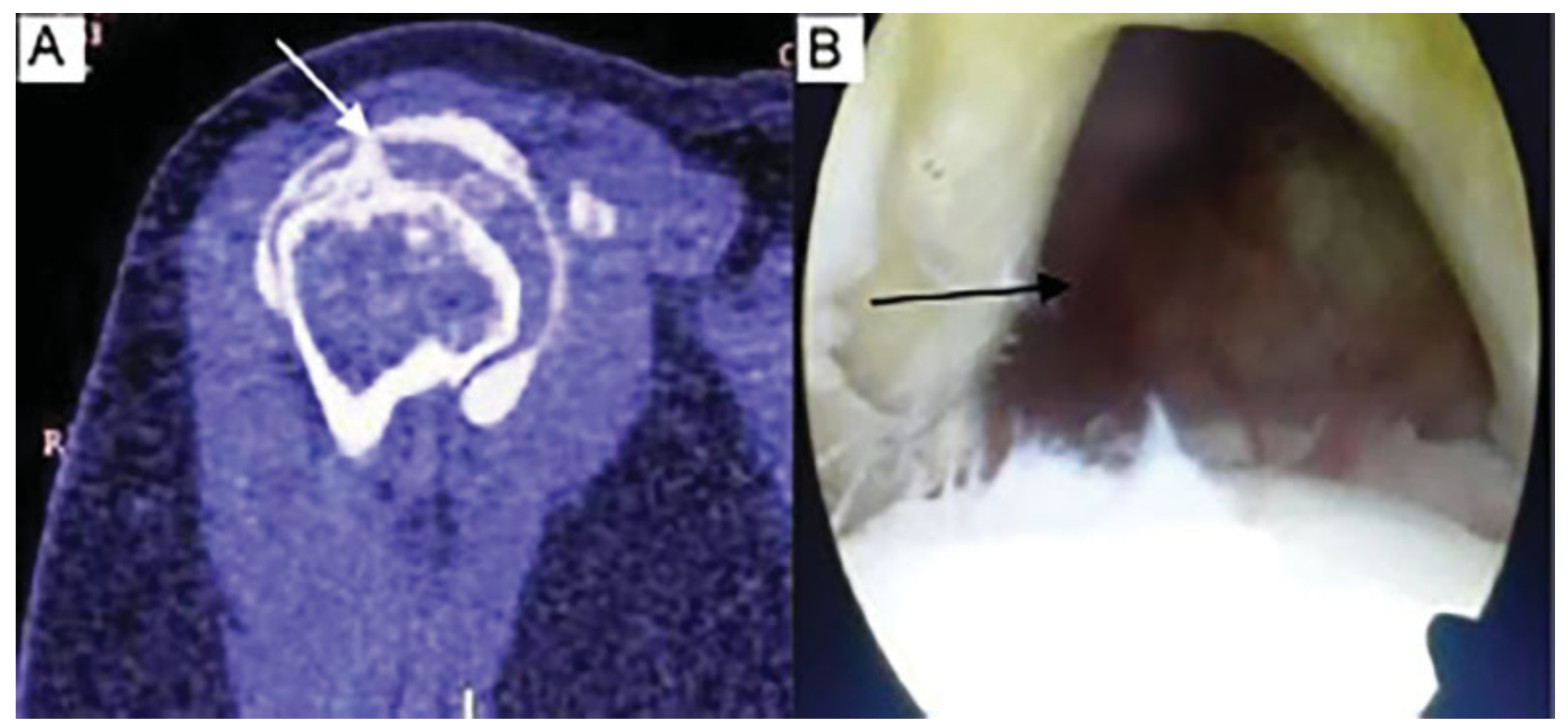

Fig. 2 Lesão transfixante do tendão supraespinal. A, corte sagital da ATCMS (seta); B, visão artroscópica intra-articular (seta). 


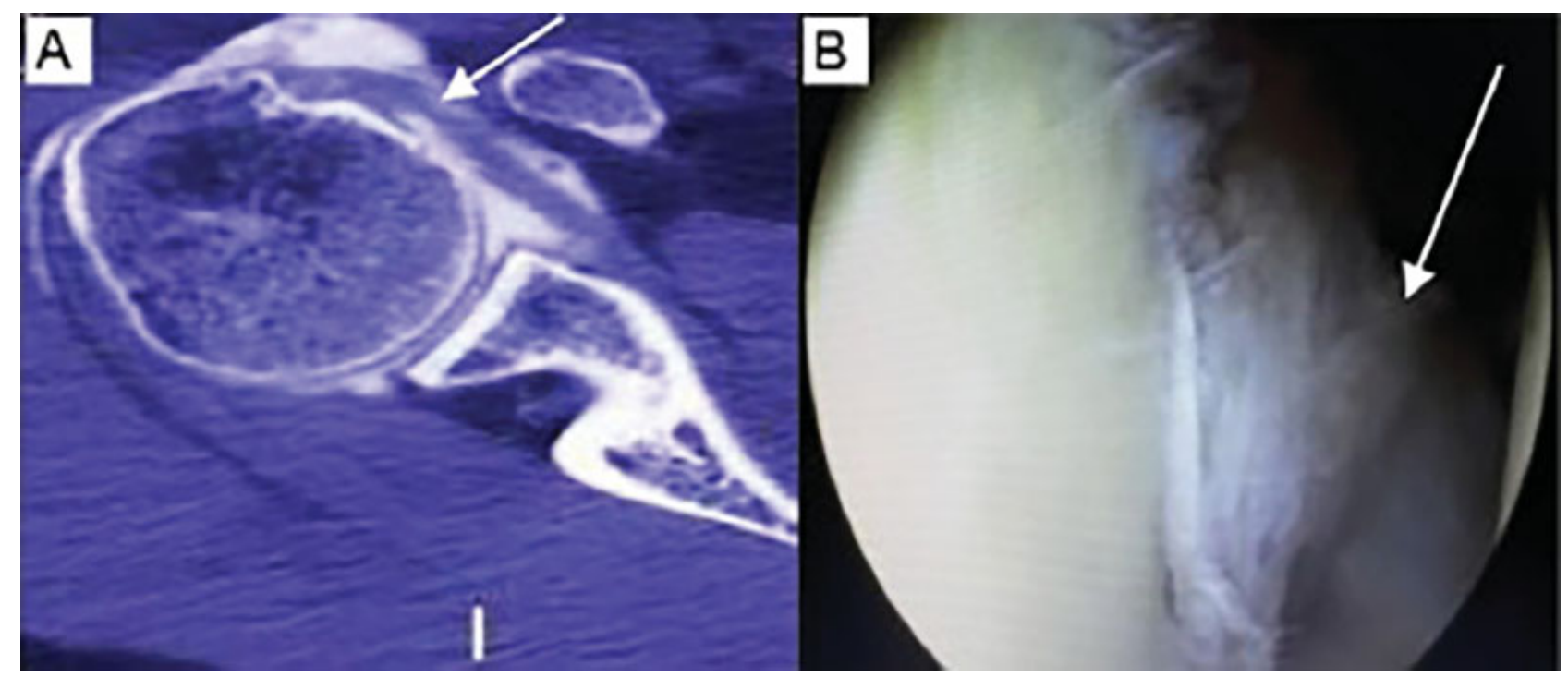

Fig. 3 Lesão parcial do tendão subescapular. A, corte axial da ATCMS (seta); B, visão artroscópica intra-articular (seta).

\section{Discussão}

Há um número reduzido de estudos científicos que comparam a ATCMS e a artroscopia na avaliação das lesões do manguito rotador, o que dificulta a correlação dos achados. Dessa forma, buscamos como opção revisar diversos estudos que discutiram algumas estruturas anatômicas correlacionadas com este estudo.

As lesões do manguito rotador têm uma incidência desconhecida na população em geral, com uma prevalência alta, que aumenta de acordo com idade e que, por volta de 60 anos, alcança uma incidência próxima a $60 \%{ }^{6,12}$ No nosso estudo, a idade média dos pacientes foi de 57 anos.

O membro dominante é o mais frequentemente acometido nas lesões do manguito rotador, provavelmente em função da sua maior demanda quando comparado com o lado oposto. ${ }^{13}$ Observamos em nosso estudo que $60 \%$ ( 18 pacientes) dos casos acometeram o lado dominante do paciente, o que corrobora os achados da literatura.

Apesar dos nossos pacientes cursarem com um número significativo de comorbidades, assim como nos estudos na literatura, essas não foram consideradas um fator negativo na indicação do procedimento cirúrgico e sua subsequente reabilitação pós-operatória. ${ }^{14,15}$

Quanto a outras lesões (lesão labral, cartilaginosa, óssea, do cabo longo do tendão do bíceps) associadas às lesões do manguito rotador, observamos no nosso estudo que a ATCMS foi capaz de identificá-las com a mesma precisão da artroscopia. Muitos autores entendem que a lesão do manguito rotador e a instabilidade com lesões associadas estão estreitamente relacionadas, principalmente em indivíduos mais velhos, com mais de 60 anos. ${ }^{16-19}$ Apesar de não haver entre os pacientes estudados casos de instabilidade do ombro associada, lesões associadas foram identificadas.

Mesmo com todos os avanços alcançados no campo da imagiologia musculoesquelética, até o presente momento não há um consenso estabelecido a respeito de qual técnica de imagem fornece os melhores resultados no diagnóstico, e sobre o acompanhamento dos pacientes com lesão do manguito rotador do ombro; tampouco há uma padronização de metodologias disponíveis caso não seja possível o exame de RM, que é considerado, hoje em dia, o melhor método de imagem para o diagnóstico de lesões do manguito rotador do ombro.

No entanto, sabe-se que a artrotomografia computadorizada (ATC) e a artrorressonância magnética (ARM) são exames frequentemente usados na avaliação pré-operatória das lesões do manguito rotador. ${ }^{20-22}$

A vantagem da ARM sobre a RM convencional é que o líquido do contraste introduzido na articulação a distende, permite separar as estruturas, e promove um melhor detalhamento das estruturas intracapsulares do ombro e melhor apreciação da sua anatomia complexa e das suas variações anatômicas; além disso, o meio de contraste realça as estruturas da articulação e, consequentemente, torna as lesões mais nítidas. Uma desvantagem é transformar um exame não invasivo em exame minimamente invasivo, mas que é universalmente tolerado. ${ }^{23}$

Farley et $\mathrm{al}^{24}$ em estudo comparativo, avaliaram, pela ARM, 36 pacientes com lesões parciais ou completas do manguito rotador confirmadas por via artroscópica. Com base na observação radiológica, o diagnóstico foi verdadeiro positivo em 12 pacientes, verdadeiro negativo em 16, falso positivo em 3 , e falso negativo em 5 . A sensibilidade isolada da ARM foi de $71 \%$, a especificidade, de $84 \%$, e a acurácia, de 78\%. No nosso estudo, com base na ATCMS, observamos achados com um melhor resultado, com uma sensibilidade de $79,41 \%$, especificidade de $100 \%$, e acurácia de $81,57 \%$.

De acordo com Rhee et al, ${ }^{25}$ o uso combinado da artrografia do ombro com a RM e a TC oferece vantagens distintas com relação às imagens convencionais (sem o uso do meio de contraste articular). O meio de contraste e a distensão articular aprimoraram a avaliação de várias estruturas articulares, e ajudaram a distinguir anormalidades sutis e variantes anatômicas normais. Entendemos que essa vantagem permite uma melhor definição diagnóstica, e possibilita um planejamento cirúrgico mais acertado. 
Como é uma modalidade de imagem relativamente nova, a ATCMS também pode demonstrar alterações na cartilagem articular nos ombros, por meio de cortes finos e da produção de alto fluxo de fótons (200-750 mAs). O resultado é a possibilidade de obtenção de imagens diagnósticas, multiplanares e reconstruções tridimensionais do ombro.

Apesar de ser considerada o exame de imagem padrãoouro na avaliação do manguito rotador, a RM pode ser contraindicada em algumas situações, como, na presença de marca-passo, claustrofobia, e artefatos metálicos. Nossa observação é corroborada também por outros autores, que entendem que a ATC com multidetector é uma opção válida para os pacientes com contraindicação à RM. ${ }^{26,27}$

Charousset et al $^{28}$ confirmam o valor da ATC no diagnóstico das lesões do manguito rotador, e observam sensibilidade e especificidade consideráveis das lesões dos tendões dos músculos supraespinal e infraespinal. No nosso trabalho, alcançamos um especificidade de $100 \%$, e uma sensibilidade de $79,41 \%$ nas lesões do manguito rotador de um modo geral.Já nas lesões dos tendões supraespinal e infraespinal, observamos uma sensibilidade de 78,78\% e 78,95\%, respectivamente. Além disso, a ATCMS tem mostrado vantagens no pós-operatório, como a não produção de artefato significativo na presença de material metálico cirúrgico. ${ }^{29-32}$ Em certos casos, a presença de metal dentro ou em volta da articulação do ombro pode limitar a efetividade da RM convencional ou da ARM, a despeito das técnicas que podem aprimorar a RM. ${ }^{33-36}$

A ATCMS de alta resolução do ombro permite a visualização e o diagnóstico das lesões articulares do manguito rotador. No entanto, quanto às lesões parciais (bursais, intersticiais), 0 exame não se mostrou eficaz. ${ }^{37,38}$ Particularmente no nosso estudo, percebemos também uma maior dificuldade no diagnóstico das lesões parciais. Essa impressão foi confirmada quando, na nossa avaliação, identificamos que, enquanto a lesão parcial do tendão supraespinal foi identificada em apenas 3,33\% dos casos pela ATCMS, na artroscopia verificamos a presença desse tipo de lesão em 16,7\% dos casos.

Nas lesões completas do manguito rotador (de toda a sua espessura), os valores encontrados quanto à sensibilidade, especificidade, valores preditivos e acurácia são tão altos na ATCMS quanto na ARM. No entanto, a sensibilidade para as lesões parciais é muito baixa. ${ }^{39,40}$ No presente estudo, a acurácia da ATCMS no diagnóstico das lesões do manguito rotador $(81,57 \%)$, de um modo geral, se mostrou com um grau de concordância moderado (coeficiente Kappa). No entanto, ao avaliar a acurácia da ATCMS com especificação do tendão acometido separadamente, observamos um resultado ainda melhor, com um grau de concordância ainda maior: a acurácia na lesão do tendão supraespinal foi de $81,57 \%$; na lesão do tendão infraespinal, foi de $89,47 \%$; e na lesão do tendão subescapular, foi de $92,11 \%$. Foram obtidos graus de concordância de 49,51\% (moderado), 78,94\% (substancial), e $83,39 \%$ (quase perfeito), respectivamente.

Vale ressaltar que a ATCMS tem a grande vantagem de ser um procedimento de menor custo e de rápida realização, com menor desconforto para o paciente quando comparado com o exame de ressonância magnética.
Esta pesquisa apresenta algumas limitações. Mesmo considerada o exame padrão-ouro, a artroscopia é um método que depende do operador-apesar de o médico ortopedista ter mais de 10 anos de experiência em cirurgia de ombro por via artroscópica. Outro viés foi o fato de que neste estudo todos os pacientes operados apresentavam lesão do manguito rotador, o que comprometeu os achados da especificidade, que, no nosso trabalho, foi de 100\%. Para minimizar essa limitação, optamos por identificar um grupo em nossos dados históricos que tivesse resultado negativo para a lesão do manguito rotador na avaliação artroscópica, mas que tivesse sido diagnosticado na avaliação radiológica pela ATCMS, considerado um grupochave na avaliação e determinação dos achados estatísticos.

\section{Conclusão}

Diante da impossibilidade de relização da RM (exame de imagem padrão-ouro), a ATCMS se mostra um exame de imagem capaz de avaliar/diagnosticar as lesões do manguito rotador. Com isso, o médico assistente tem condições melhores de o planejar sua estratégia de abordagem terapêutica.

Conflitos de Interesse

Os autores declaram não haver conflitos de interesse.

\section{Referências}

1 Ellman H, Kay SP. Arthroscopic subacromial decompression for chronic impingement. Two- to five-year results. J Bone Joint Surg Br 1991;73(03):395-398

2 Veado MAC, Fonseca RMF. O ombro do nadador veterano. Rev Bras Ortop 1992;27:686-690

3 Farin PU, Kaukanen E, Jaroma H, Väätäinen U, Miettinen H, Soimakallio S. Site and size of rotator-cuff tear. Findings at ultrasound, double-contrast arthrography, and computed tomography arthrography with surgical correlation. Invest Radiol 1996;31(07):387-394

4 Waldt S, Bruegel M, Mueller D, et al. Rotator cuff tears: assessment with MR arthrography in 275 patients with arthroscopic correlation. Eur Radiol 2007;17(02):491-498

5 Meislin RJ, Sperling JW, Stitik TP. Persistent shoulder pain: epidemiology, pathophysiology, and diagnosis. Am J Orthop 2005;34)12, Suppl)5-9

6 Andrade RP, Filho MRCC, Queiroz BC. Lesões do manguito rotador. Rev Bras Ortop 2004;39(11/12):621-636

7 Bedi A, Dines J, Warren RF, Dines DM. Massive tears of the rotator cuff. J Bone Joint Surg Am 2010;92(09):1894-1908

8 Matava MJ, Purcell DB, Rudzki JR. Partial-thickness rotator cuff tears. Am J Sports Med 2005;33(09):1405-1417

9 Lech O, Valenzuela Neto C, Severo A. Tratamento conservador das lesões parciais e completas do manguito rotador. Acta Ortop Bras 2000;8(03):144-156

10 McLaughlin HL. Rupture of the rotator cuff. J Bone Joint Surg Am 1962;44:979-983

11 Landis JR, Koch GG. The measurement of observer agreement for categorical data. Biometrics 1977;33(01):159-174

12 Guimarães MV. Avaliação do tratamento conservador do pinçamento subacromial e das lesões do manguito rotador. Rev Bras Ortop 1995;30(09):645-648

13 Almeida A, Valin MR, Zampieri R, Almeida NC, Roveda G, Agostini AP. Análise comparativa do resultado da sutura artroscópica da lesão do manguito rotador em pacientes fumantes e não fumantes. Rev Bras Ortop 2011;46(02):172-175 
14 Checchia SL, Santos PD, Volpe Neto F, Cury RPL. Tratamento cirúrgico das lesões completas do manguito rotador. Rev Bras Ortop 1994;29(11/12):827-836

15 Tashjian RZ, Henn RF, Kang L, Green A. The effect of comorbidity on self-assessed function in patients with a chronic rotator cuff tear. J Bone Joint Surg Am 2004;86(02):355-362

16 Porcellini G, Paladini P, Campi F, Paganelli M. Shoulder instability and related rotator cuff tears: arthroscopic findings and treatment in patients aged 40 to 60 years. Arthroscopy 2006;22(03): 270-276

17 Mileski RA, Snyder SJ. Superior labral lesions in the shoulder: pathoanatomy and surgical management. J Am Acad Orthop Surg 1998;6(02):121-131

18 Voos JE, Pearle AD, Mattern CJ, Cordasco FA, Allen AA, Warren RF. Outcomes of combined arthroscopic rotator cuff and labral repair. Am J Sports Med 2007;35(07):1174-1179

19 Shin SJ, Yoo JC, McGarry MH, Jun BJ, Lee TQ. Anterior capsulolabral lesions combined with supraspinatus tendon tears: biomechanical effects of the pathologic condition and repair in human cadaveric shoulders. Arthroscopy 2013;29(09):1492-1497

20 Boissonnault WG, Badke MB, Wooden MJ, Ekedahl S, Fly K. Patient outcome following rehabilitation for rotator cuff repair surgery: the impact of selected medical comorbidities. J Orthop Sports Phys Ther 2007;37(06):312-319

21 de Jesus JO, Parker L, Frangos AJ, Nazarian LN. Accuracy of MRI, MR arthrography, and ultrasound in the diagnosis of rotator cuff tears: a meta-analysis. AJR Am J Roentgenol 2009;192(06):1701-1707

22 Chung CB, Corrente L, Resnick D. MR arthrography of the shoulder. Magn Reson Imaging Clin N Am 2004;12(01):25-38, v-vi

23 Gómez GFCA. Estudo comparativo entre artrotomografia computadorizada multislice e artrorressonância magnética na instabilidade do ombro correlacionadas com os achados artroscópicos São Paulo: Departamento de Radiologia, Faculdade de Medicina da Universidade de São Paulo; 2008

24 Farley TE, Neumann CH, Steinbach LS, Jahnke AJ, Petersen SS. Fullthickness tears of the rotator cuff of the shoulder: diagnosis with MR imaging. AJR Am J Roentgenol 1992;158(02):347-351

25 Rhee RB, Chan KK, Lieu JG, Kim BS, Steinbach LSMR. MR and CT arthrography of the shoulder. Semin Musculoskelet Radiol 2012; 16(01):3-14

26 Fritz J, Fishman EK, Small KM, et al. MDCT arthrography of the shoulder with datasets of isotropic resolution: indications, technique, and applications. AJR Am J Roentgenol 2012;198(03):635-646
27 De Filippo M, Bertellini A, Sverzellati N, et al. Multidetector computed tomography arthrography of the shoulder: diagnostic accuracy and indications. Acta Radiol 2008;49(05):540-549

28 Charousset C, Bellaïche L, Duranthon LD, Grimberg J. Accuracy of CT arthrography in the assessment of tears of the rotator cuff. J Bone Joint Surg Br 2005;87(06):824-828

29 Rydberg J, Buckwalter KA, Caldemeyer KS, et al. Multisection CT: scanning techniques and clinical applications. Radiographics 2000;20(06):1787-1806

30 Penrod B, Lane M, Hayda R, Tucker J, Prevost D, DeBerardino T. Multislice/multi-detector et for the evaluation of orthopaedic appliances: utility and optimization in a variety of clinical conditions. In: Society of Skeletal Radiology. Twenty-fourth Annual Meeting. 2001

31 Farber JM, Buckwalter K. Multislice CT and the evaluation of osseous structures and joints in the presence of metal. In: Society of Skeletal Radiology. Twenty-fourth Annual Meeting. 2001

32 Farber JM, Buckwalter KA. Sports-related injuries of the shoulder: instability. Radiol Clin North Am 2002;40(02):235-249

33 Owen RS, Iannotti JP, Kneeland JB, Dalinka MK, Deren JA, Oleaga L. Shoulder after surgery: MR imaging with surgical validation. Radiology 1993;186(02):443-447

34 Rand T, Trattnig S, Breitenseher M, Freilinger W, Cochole M, Imhof H. [MR arthrography of the shoulder joint in a postoperative patient sample]. Radiologe 1996;36(12):966-970

35 Magee TH, Gaenslen ES, Seitz R, Hinson GA, Wetzel LH. MR imaging of the shoulder after surgery. AJR Am J Roentgenol 1997;168(04):925-928

36 Gusmer PB, Potter HG, Donovan WD, O'Brien SJ. MR imaging of the shoulder after rotator cuff repair. AJR Am J Roentgenol 1997;168 (02):559-563

37 Omoumi P, Bafort AC, Dubuc JE, Malghem J, Vande Berg BC, Lecouvet FE. Evaluation of rotator cuff tendon tears: comparison of multidetector CT arthrography and 1.5-T MR arthrography. Radiology 2012;264(03):812-822

38 Fritz J, Fishman EK, Fayad LM. MDCT Arthrography of the Shoulder. Semin Musculoskelet Radiol 2014;18(04):343-351

$39 \mathrm{Oh} \mathrm{JH}$, Kim JY, Choi JA, Kim WS. Effectiveness of multidetector computed tomography arthrography for the diagnosis of shoulder pathology: comparison with magnetic resonance imaging with arthroscopic correlation.J Shoulder Elbow Surg 2010;19(01):14-20

40 Mahmoud MK, Badran YM, Zaki HG, Ali AH. One-shot MR and MDCT arthrography of shoulder lesions with arthroscopic correlation. Egyptian J Radiol Nuclear Med. 2013;44:273-281 